\title{
Active Fractions of Methanol Crude Obtained from Acacia seyal gum: Antioxidant Capacity using FTIR Analysis
}

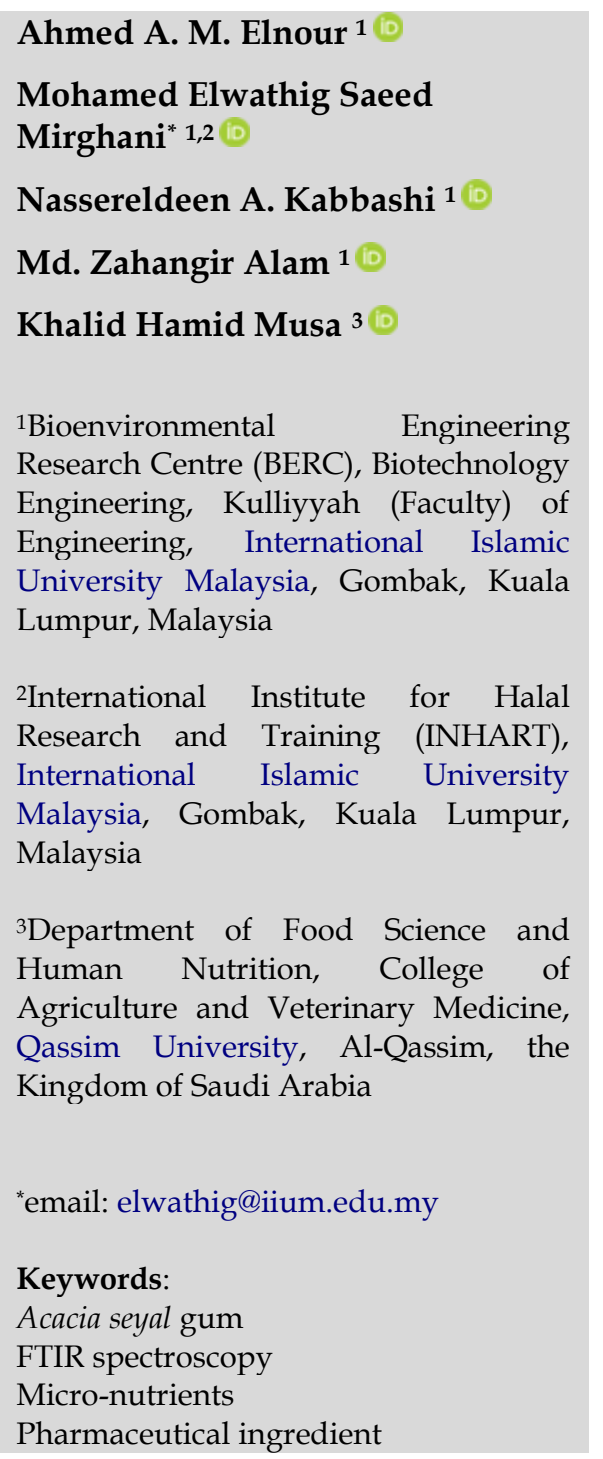

\begin{abstract}
The present study is on Acacia seyal gum (ASG), which is an exudate from the Talha tree. It provides a rich source of polyphenolics compounds that are used traditionally in folk medicine. The study aims to determine the antioxidant capacity (AC) and functional groups of ASG and Prebio-T-commercial (PTC) samples. The methanol crude extracts of both ASG and PTC have fractioned into chloroform (CHF), hexane (HXF), acetone (AF), and methanol (MF) using solvent-solvent portion. Both ferric reducing antioxidant power (FRAP) and cupric reducing antioxidant capacity (CUPRAC) assays for each fraction examined. Crude methanol extracts (CME) and its active compositions also analyzed carefully using Fourier Transform Infrared Spectroscopy (FTIR) technique. The findings presented a wide variety of functional groups provided by the FTIR spectra (eight bands approximately). Regarding cupric reducing antioxidant capacity (CUPRAC), the methanol crude extracts values are $888.6 \pm 4.57 \mathrm{mg}$ TE $/ 100 \mathrm{~g}$ extract, for PTC as compared to $474.3 \pm 2.23 \mathrm{mg}$ TE $/ 100 \mathrm{~g}$ of extract for ASG. However, both methanol and acetone fractions revealed significantly ( $\mathrm{p} \leq 0.05$ ) high FRAP values ranged between $599.8 \pm 7.5$ and $741.8 \pm 5.8 \mathrm{mg}$ TE/100g fraction; for PTC and ASG, respectively. While CUPRAC showed insignificant $(\mathrm{p} \geq 0.05)$ same values 356.1 $\pm 2.62 \mathrm{mg}$ TE/100g of fraction; for MF of both PTC and ASG, respectively. Therefore, in this study, MFs are found to be more effective than acetone fractions AFs, except for CHF and HXF. Finally, the antioxidant activity of the active fraction has provided some evidence regarding its functional groups, which may have used in traditional medicine as well as the prevention of degenerative disease. Based on this study, the AC of CME and its precious fractions considered promising and beneficial for using ASG in food processing, medicine, and pharmaceutical industries.
\end{abstract}

Received: August 16 2019

Accepted: October 22nd 2019

Published: November 14th 2019

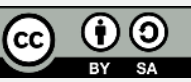

(c) 2019 Ahmed A M Elnour, Mohamed Elwathig Saeed Mirghani, Nassereldeen A Kabbashi, Md Zahangir Alam, Khalid Hamid Musa. Published by Institute for Research and Community Services Universitas Muhammadiyah Palangkaraya. This is an Open Access article under the CC-BY-SA License (http://creativecommons.org/licenses/by-sa/4.0/). DOI: https://doi.org/10.33084/bjop.v2i2.915.

\section{INTRODUCTION}

Gum arabic (GA) is a dried exudate nutritionally valuable, rich in dietary fibers, and polyphenolics compounds which support healthy living due to the antioxidant properties, or as food ingredients in a wide variety as a pharmaceutical. Gum Arabic is one of the world's major natural commodities of commerce and constitutes a valuable export commodity in countries such as Niger, Chad, Senegal, Nigeria, and especially Sudan, which controls about $60-85 \%$ of the world's export of GA (Anderson, 1993). Tones of GA are exported annually and has been estimated to the tune of US\$ 337 million per year in 2014-2016, 44\% of which accrued to 
raw materials and semi-processed gum and $56 \%$ to processed gum (Magid et al., 2014). Thus, GA has an economically beneficial positive impact on the human lifestyle. The analysis of GA showed that the substance is acceptable for human daily intake by the Joint Expert Committee on Food Additives (JECFA) since 1969 (Musa et al., 2015). Therefore, in Sudan and worldwide, they are exploited mainly for food and industrial production. Its first industrial applications like confectionery, beverages, flavor encapsulators, ceramics), and health aspects (Magid et al., 2014). Gum Arabic is the excellent source of bioactive compounds such as dietary fiber, protein, essential amino acids, minerals and vitamins (Chawla \& Patil, 2010) and they contain many biologically active substances like proteins, polysaccharides, and polyphenols (Elnour et al., 2018). Thus, GA recommended for the primary source of health functional foods.

Antioxidants are offering to see that essential useful food, and thus in biological systems have multiple capabilities, which include protecting against oxidative destruction in the critical singling pathways of cellular material. Many human-made antioxidants (SA), including butylated hydroxyl toluene (BHT), butylated hydroxyanisole (BHA), and tertbutyl hydroquinone (TBHQ) will be commercially available and therefore are at present used meant for foodstuff applications (Jayasri \& Narayanan, 2007). Even so, their use is today constrained due to their unwanted side effects. It has proved that they encourage the development of malignant skin cells in mice. These kinds of effects have got strong the development of the initiative signed to produce other antioxidants by an organic material just like GA (Godic et al., 2014) Within the last many years, GA using raw materials have explained that they can have the biological activity of potential healing benefit (Minzanova et al., 2018). A study was conducted by Glover et al. (2009) to evaluate the cardiovascular and renal effects of supplementation with $25 \mathrm{~g} /$ day of GA in healthy individuals and those with diabetic nephropathy for up to 12 weeks. Mean systolic blood pressure reduced in response to GA supplementation. Another study also reported the effectiveness of dietary supplementation with GA on blood pressure, which was observed in participants with diabetes and mild renal involvement as well as in a general healthy normotensive cohort. The results support previous data about the safety and tolerance of GA in humans and demonstrate that GA used by diabetic subjects with significant renal impairment (Doi et al., 2006). Another study by Al-Mosawi (2002) was conducted to investigate the use of GA in children with renal failure. Gum arabic effects on blood pressure, blood urea concentrations, and the frequency of intermittent peritoneal dialysis (IPD) observed. Gum arabic is given for 48 days; the dose was $0.5 \mathrm{~g} / \mathrm{kg}$ per day in two to three divided doses, and the dose was diluted with a minimal amount of water to make it acceptable. The main results indicate that GA showed a beneficial antihypertensive effect.

Consequently, new curiosity has been created to search organic and secure phenolic compounds coming from GA, which usually are regarded as a supply of bioactive substances since they can make a significant number of secondary metabolites and, a broad range of natural activities characterizes them. Substances with antihypertension, antidiabetics, and also prebiotic properties have recently recognized in GA (Rehman et al., 2003), and it should use for their multipurpose homes in the type of meals, energy, medication, and makeup products and while biotechnological equipment (Ali et al., 2013a). Therefore, GA includes a unique chemical substance structure that might have a crucial bioactive compound. 
Chemically, GA is a complex mixture of macromolecules of different sizes and composition - mainly carbohydrates and proteins. It is rich in non-viscous soluble fibers with high dietary value, and also contains minerals like potassium, magnesium, and calcium (Ali et al., 2013a; Elnour, 2007). Gum arabic antioxidant properties, nephroprotectant, and other effects have been highlighted in recent studies (Ali et al., 2013b). Its role in the metabolism of lipids (Tiss et al., 2001) and its positive effect in the treatment of several degenerative diseases such as kidney failure (Ali et al., 2013b), cardiovascular (Glover et al., 2009) and gastrointestinal diseases (Rehman et al., 2003) have also been reported. Gum arabic, therefore, promises many benefits in medical, food, and pharmaceutical industries. In this regard, some of the main challenges of better understanding GA interfacial function, as well as the role of minor components (polyphenols), seem to have been entirely neglected during the last past years (Sanchez et al., 2018). Hence, further analytical techniques such as Fourier transform infrared spectroscopy (FTIR) is necessary for antioxidant extraction from GA and characterization.

Fourier transform infrared spectroscopy is one of the most frequently used methods in identifying chemical substances and elucidating the compound structures, where many countries utilize it as a requisite method in identifying medicines for pharmacopeia. Recently, this method has been assumed for an essential role in pharmaceutical analysis, owing to the extensive applicability and fingerprint characters over the samples (Ouhaddouch et al., 2019). The urge to modernizing the traditional medicines and the rapid global demand for bioactive compounds extraction required a fast and efficient method for analyzing the complicated mixture system. Together with the development of computer science, the development of the FTIR spectroscopic technique has done much work in the researches of bioactive contents, a traditional medicine (Reinholds et al., 2015). However, further advanced techniques for enabling FTIR to analyze the completed bioactive compounds are urgently needed. Therefore, the FTIR spectroscopy has a suitable application in assessing the quality of GA which is used mainly to determine rheology and emulsifying stability (Niu et al., 2016), as well as to discriminate the GA botanical location, type, and age of the tree (Wang et al., 2014). Thus, FTIR is a hugely affected tool for the characterization of antioxidant compounds. In this study, FTIR spectroscopy has applied in the prediction of antioxidant activity (AC) and phenolic compounds of Acacia seyal gum. Therefore, the development of accurate, rapid, and feasible analysis methods which integrally reflecting the inherent qualities of medicinal materials and their corresponding extracts is required. This should involve the crude methanol extract (CME) antioxidant capacities and their fractions from ASG natural exudate and commercial product of Prebio$\mathrm{T}$ using free radical scavenging.

\section{MATERIALS AND METHODS}

\section{Chemicals}

The chemicals and reagents used in the study include; ferric chloride $\left(\mathrm{FeCl}_{3} 6 \mathrm{H}_{2} \mathrm{O}\right)$ and $\mathrm{HCl}$ obtained from Merck ${ }^{\circledR}$. Others include 2, 4, 6-tris (2-pyridyl)-s-triazine (TPTZ), Neocuproine (2,9-Dimethyl-1,10 phenanthrolines), Trolox, Sodium and Ammonium acetate trihydrate from Sigma ${ }^{\circledR}$. Sodium acetate RDH (Germany) and glacial acetic acid from Mallinckrodt Baker $^{\circledR}$ have used. All chemicals and reagents were of analytical grade. Spectrophotometric measurements were done using Spectro Star Nano spectrophotometer with microplate, which contained 96 micro cuvettes.

\section{Plants materials}

Acacia seyal gum was collected from the Blue NileState of Sudan. Samples are correctly identified and cleaned from 
plant bark parts and sand. In direction to, make the homogeneity of the samples, and random selection of nodules was grounding and made into a mechanical powder using US standard testing sieve (Fisher Company) with $1.40 \mathrm{~mm}$ mish size. For ASG commercial sample called Prebio -T (PTC) was obtained from Perfect Life Food $^{\circledR}$ containing Homogenized Ingredients. Finally, partitioning modules were used according to solvent-solvent partitioning (SSP) methods by Kupchan et al. (1969), with slights modification by Elnour et al. (2018). The CME of both ASG and PTC being used as a sample. Extract preparation and SSP schematic flow were presented as described in Figure 1.

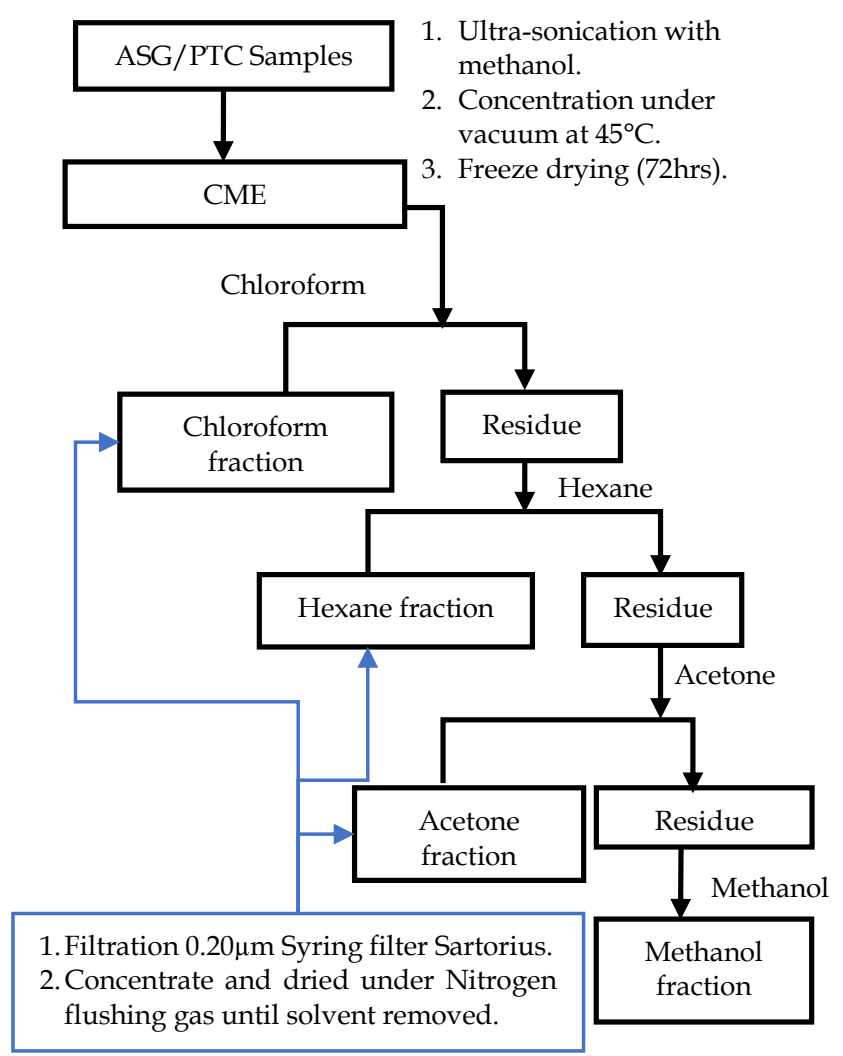

Figure 1. Schematic flow representation of the Kupchan SSP of a CME of ASG and PTC and its fractions

\section{Antioxidant activity determination}

Cupric reducing antioxidant capacity

The procedure of the Cupric reducing antioxidant capacity (CUPRAC) method is explained by Apak et al. (2008), with slight modification. Briefly, $1 \mathrm{ml}$, each, of
Copper (II) chloride, neocuproine (2, 9-dimethyl-1, 10phenanthroline), ammonium acetate buffer, and water has mixed before adding with the sample $(0.1 \mathrm{ml})$. After 30 minutes, the absorbance recorded at $450 \mathrm{~nm}$ of wavelength against a reagent blank using the UV-Vis Nanostar-spectrophotometer SN:1510-01734C, type 1510 used. The result has presented as $\mathrm{mg}$ of Trolox Equivalent (TE) per $100 \mathrm{~g}$ of dry sample (mg TE/100 $\mathrm{g}$ of DW). Methanol solutions of known Trolox concentrations in the range of 0 to $100 \mu \mathrm{g} / \mu \mathrm{l}$ used for the calibration curve, and the results were expressed as mg TE/100 g of DW.

\section{Determination offerric reducing antioxidant power}

A working fresh ferric reducing antioxidant power (FRAP) reagent prepared as described by Musa et al. (2013) with some slight modification. The working reagent formulated using mixing $300 \mathrm{~mm}$ acetate buffer having pH 3.6 (constituted by $3.1 \mathrm{~g}$ sodium acetate trihydrate plus $16 \mathrm{ml}$ glacial acid) with distilled water in a ratio 1 : 1; plus $10 \mathrm{~mm}$ TPTZ, in $40 \mathrm{~mm} \mathrm{HCl}$; and with $20 \mathrm{~mm} \mathrm{FeCl} \cdot 6 \mathrm{H}_{2} \mathrm{O}$ in the ratio of $10: 1: 1$. After 30 minutes, about $1 \mathrm{ml}$ of FRAP reagent adds to $100 \mu \mathrm{l}$ of samples. By using a UV-Vis Nanostarspectrophotometer SN:1510-01734C, type 1510 placed at $595 \mathrm{~nm}$ wavelength, and the experimental result presented in mg of TE per $100 \mathrm{~g}$ of fresh sample (mg TE/100 $\mathrm{g}$ of DW).

\section{FTIR Spectroscopy}

The infrared (IR) spectra were recorded using the FTIR spectrometer (Nexus 670 Fourier Transform Infrared spectrometer, Thermo Nicolet), and the spectra were analyzed using Omnic 5.2a software. Dry nitrogen was used to purge the instrument with automatic dehumidifiers preventing the interference of $\mathrm{CO}_{2}$ and water vapor from entering the sample chamber. The prepared samples deposited on top of the attenuated total reflectance (ATR) element that has been previously 
rinsed with acetone three times and rubbed with a soft tissue to dry. The cleaned ATR accessory was checked spectrally after every five measurements, ensuring its cleanliness from previous sample residues (Lebon et al., 2014). FTIR spectra of CME, with its active methanol fraction $(\mathrm{MF})$ and acetone fraction $(\mathrm{AF})$, were acquired through strong apodization over the frequency of 4000$400 \mathrm{~cm}^{-1}$ in the co-addiction of 32 scans at $1 \mathrm{~cm}^{-1}$ resolution and a gain of 2.0. Sample spectra were measured against the blank (air) spectrum. From each prepared sample, two spectra were collected and stored at the Joint Committee on Atomic and Molecular Physical Data Data Exchange (JCAMP) for ensuing analysis.

\section{Statistical analysis}

Each assay of the AC was conducted three times using the same extract. Analysis of variance using Minitab® Software version 17 was done to determine the differences in AC for FTIR techniques. Fisher's new multiple range tests were used to determine significant differences, whereas the correlations among recorded data were calculated using Pearson's correlation coefficient (r).

\section{RESULTS AND DISCUSSION}

The yield of crude extract and solvent-partitioned fractions

Figure 2 reveals the yield of crude extract (YCE) and solvent-partitioned fractions (SPF) for raw ASG and PTC, respectively. The yield of ASG CME was found to be at $11.10 \%$, compared to the yields of PTC at $15.56 \%$. According to the best of author knowledge, there is no contemporary information about gum extraction regarding AC. However, some considerably higher yields of CME are reports from several types of plants, including Cameroonian medicinal plants, as reported by Tagne et al. (2014), who highlighted the same value of mangrove plant. However, the yield of extracts differences in ASG and PTC, respectively. This difference may be due to the variations between manufacturing techniques, processes, and extraction conditions, such as solvents fluctuations, solvent polarities, time of extraction, and temperatures.

Interestingly, the ASG yield on SPF showed an amount of $3.3 \%$ for $\mathrm{MF}$ and about $2.34 \%$ for $\mathrm{AF}$, respectively. Whereas, the PTC obtained only $3.3 \%$ and $4.21 \%$ for both $\mathrm{MF}$ and $\mathrm{AF}$, respectively. Within each sample and fractions, analysis of variance showed significant differences $(\mathrm{P} \leq 0.05)$ between the yields percentage.

Overall, the data shown in Figure 2 demonstrate that the mean value of the AF from the PTC sample was significantly ( $\mathrm{P} \leq 0.05)$ higher yields compared to that for other fractions followed by MF. This may have to the effect of some polar compounds extracted with the solvent mixture, which explained the difference in the yields. As a result, the polarity of the constituents always affects the extractants and solvent systems efficiency for fractionation and extraction techniques. The fractionation of crude extracts was carried out using both polar and non-polar solvents. To improve the efficiency of phenolics extraction, the pure chloroform, acetone, hexane, and methanol were used in the process of chlorophyll and non-polar constituent removal during the gum powder simultaneous extraction and fractionation. This attempt allowed for the optimization of the extraction condition and fractionation. Based on the results, there might be significant differences in ASG extracts from both the composition and ratio of their component that depends on the species and extraction methods used. 


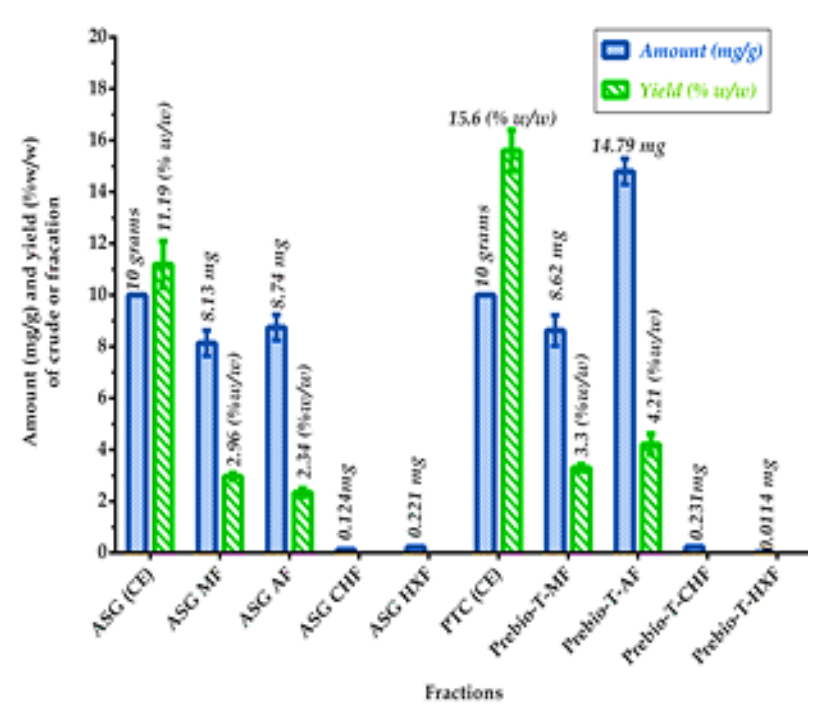

Figure 2. Presents the quantity of crude/fractions $(\mathrm{mg} / \mathrm{g})$ and yield of crude extract $(\% \mathrm{w} / \mathrm{w})$, using SPF from both raw ASG and PTC, obtained after Kupchan-partitioning of the CME and its fractions

\section{Cupric reducing antioxidant capacity}

Figure 3 shows the CUPRAC values of ASG and PTC crude extracts and different fractions, respectively. In Figure 3, the CUPRAC assay was implemented for the first time in evaluating the antioxidant activity of ASG fractions, respectively. The maximum values of CUPRAC have been estimated using CME for PTC at $888.6 \mathrm{mg}$ TE/100 g DW. In comparison with ASG CME, which was found to be at $474.3 \mathrm{mg}$ TE/100g DW. Moreover, the CUPRAC results of both MF and AF were also notably high with respective average values of MF at $599.8 \mathrm{mg}$ TE/100g DW and 741.8 mg TE/100g DW for ASG and PTC. Meanwhile, the subsequent mean value of AF at $469.8 \mathrm{mg}$ TE/100 $\mathrm{g}$ DW and 610.4 mg TE/100 $\mathrm{g}$ DW for ASG and PTC, respectively.

The CUPRAC assay results have revealed significant differences $(\mathrm{P} \leq 0.05)$ between the $\mathrm{CME}$ and its fractions, whereas they also significantly $(\mathrm{P} \leq 0.05)$ affected the antioxidant activity. However, no results calculated for chloroform fraction (CHF) and hexane fraction (HXF), which may be due to the polarity of the solvent and sample. Therefore, it has assumed that high solvent polarity increased the capacity of copper-reducing, as methanol and acetone $\mathrm{CME}$ and its fractions were found to amplify the CUPRAC value.

The results agree with Kaewseejan et al. (2015), who reported similar average CUPRAC values regarding Gynura procumbens leaves. Conclusively, it has suggested that gum methanol crude extract and its precious fractions could experience the efficacy of metal ion reducing/chelating.

\section{Ferric reducing antioxidant power}

As shown in Figure 3, the FRAP content of CME and fractions have revealed as mg Trolox equivalent/100 $\mathrm{g}$ DW). Statistically, the FRAP values were significantly (P $\leq 0.05)$ different. FRAP content was $741.8 \pm 5.81 \mathrm{mg}$ TE/100 g DW for PTC and 599.8 $\pm 7.5 \mathrm{mg}$ TE/100 g DW of ASG for all the same fraction. Results indicated a significant $(\mathrm{P} \leq 0.05)$ difference between the two fractions. The FRAP values obtained from ASG, which included four fractions consisting of $\mathrm{MF}, \mathrm{AF}, \mathrm{CHF}$, and $\mathrm{HXE}$, respectively. These fractions ranged from $469.8 \pm 10.2$ to $599.8 \pm 7.5 \mathrm{mg}$ TE/100 g DW with a mean value of 537.8 $\mathrm{mg}$ TE/100 $\mathrm{g}$ DW, whereas PTC was found to be in the range of $460.4 \pm 5.61$ to $741.8 \pm 5.81 \mathrm{mg} \mathrm{TE} / 100 \mathrm{~g}$ DW, with a mean value of $604.2 \mathrm{mg} \mathrm{TE} / 100 \mathrm{~g} \mathrm{DW}$, respectively. According to the results, crude extract and solvent partitioned fractions values have a descending order of $\mathrm{CME}>\mathrm{MF}>\mathrm{AF}$ (except CHF and HXF) for both samples.

Remarkably, both ASG and PTC samples have revealed a statistically significant difference $(\mathrm{P} \leq 0.05)$ that indicates CME had the highest antioxidant activity. However, $\mathrm{CHF}$ and HXF have negative values for both samples. Differences in the chemical compositions for each fraction might cause the characteristic values of FRAP on the various fractions. It is has observed that the FRAP value for MF was found to be at 741.8 $55.81 \mathrm{mg}$ TE/100 $\mathrm{g} \mathrm{DW}$, almost two times higher than the AF Trolox equivalent (vitamin C) as a synthetic antioxidant. Relevant to the 
FRAP values, the results seem to be agreed with the CUPRAC pattern (see Figure3), where CME was also the most active rich fraction of reductive ability. Thus, $\mathrm{CME}$ can be considered as a potentially useful source of natural antioxidants in the human body, preventing oxidative damages and advancing health protection.

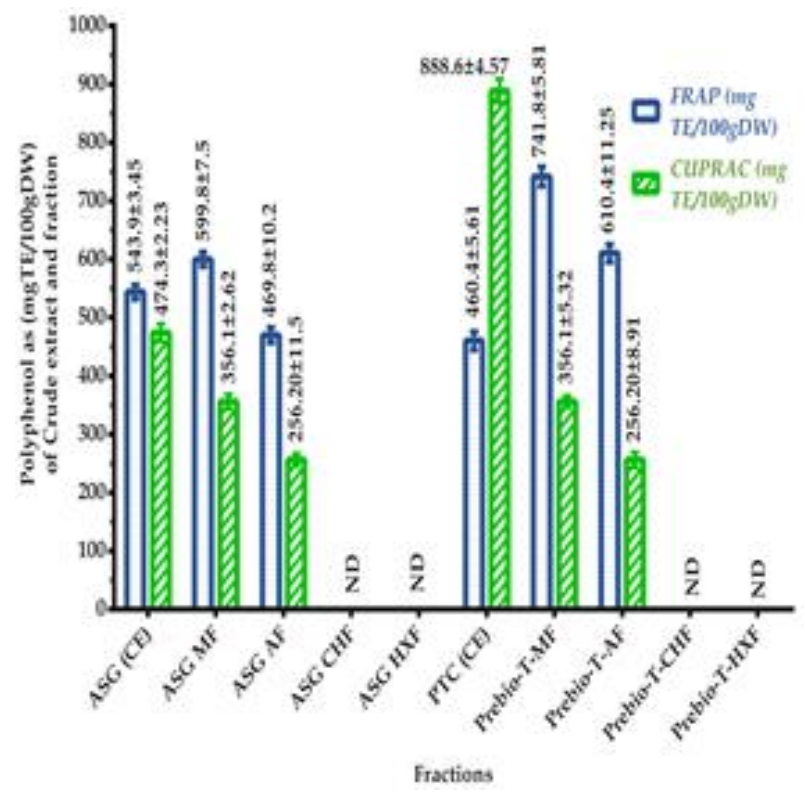

Figure 3. The antioxidant properties of four different fractions of ASG and PTC obtained after the Kupchan-partitioning of $\mathrm{CME}$ and its fractions. Results are the average of three replicates \pm Standard deviation (SD); Subscripts stars in the par charts are significantly different; *significant, and **highly significant respectively at $\mathrm{P} \leq 0.05^{* *}$ and $0.01^{* *}, \mathrm{ND}=$ not detectable.

\section{FTIR Spectroscopy}

In this paper, the FTIR spectra for both ASG and PTC regarding $\mathrm{CME}$ and active fractions are shown Figure 4 and Figure 5, respectively. Its different extracts and the extracted fractions acquired between 4000 and $400 \mathrm{~cm}^{-1}$. The resulted spectra showed some substantial overlap from each absorption spectrum of the various components, where each band provides the characteristic of absorption peaks for particular functional groups discovered from the samples. Based on Figure 4 and Figure 5 of the resulted FTIR spectra, the raw ASG and PTC (a), CME (b), and MF (c) spectra display somewhat similar patterns, where the three differ from the ranges of AF (d). The FTIR spectra of all the three enriched extracts were acquired, and a comparison made for the active peaks against the raw material gum (as a standard) (Figures $4 \mathbf{a}$ and $\mathbf{5 a}$, and $\mathbf{b}$ ). Thus, FTIR can possess excellent characterization.

The analysis of raw ASG and PTC was carried out in the solid-state to evade the significant contribution of water absorption, particularly in the band region of amide I (1720 to $1580 \mathrm{~cm}^{-1}$ ), as described by Renard et al. (2006). Sequentially, the FTIR spectrum of both samples of ASG and PTC as raw gum had obtained ten significant peaks at the approximately similar range of $418,670,1036,1256$, 1410, 1641, 2360, 2914, 3386 and $3743 \mathrm{~cm}^{-1}$ with a tiny difference in 1256 and $1363 \mathrm{~cm}^{-1}$. Three leading spectral bands displayed from both ASG and PTC in the wavenumber region connecting 1700 to $1200 \mathrm{~cm}^{-1}$ (Figures $4 \mathbf{a}$ and $\mathbf{5 a}$, and $\mathbf{b}$, bands $A$ ). The attribution of the bands may be partially related to the contribution of proteins in Acacia gums. Based on the literature for proteins FTIR spectra, the amide I and amide II vibration bands are presumed to be nearly 1650 and $1550 \mathrm{~cm}^{-1}$. The bands are presumed to be predominantly dependent on the polypeptide backbone secondary structure and rarely affected by the features of the side chains (Barth \& Zscherp, 2002). Therefore, GA has a unique in polyphenolics bands with different vibration phenomena.

However, Vinod et al. (2010) have reported that the generation of some bands in the similar wavenumber region towards 1740, 1641, and $1410 \mathrm{~cm}^{-1}$ also shown from the FTIR spectra of specific polysaccharides that carry uronic acids. Thus, the movement of the band position near $1610 \mathrm{~cm}^{-1}$ was based on the biochemical composition and specifically the uronic acid content. Also, it is has reported that the absorbance band of Arabino galactoprotein (AGPs) at the carboxylic functions diversified from 1640 to $1600 \mathrm{~cm}^{-1}$ at the increase of uronic acid content from 3.6 to 14.7\% (Boulet 
et al., 2007). Hence, further studies have needed to assess the specific functional groups in GA.

The raw ASG and PTC were composed of glucuronic acid content in a significant amount, $10.4 \%$ molar of 4-Oglucuronic acid, and low content of proteins at $1.0 \%$. Therefore, the assignment of the absorbance bands at 1602 and $1416 \mathrm{~cm}^{-1}$ in the ASG spectrum have mainly assigned to the optical density of the deprotonated carboxylic function from glucuronic acid (Manrique \& Lajolo, 2002). Furthermore, IR bands near 1370 and 1243 $\mathrm{cm}^{-1}$ in the ASG spectrum might be related to the stretching of C-O-C and the bending vibrations, as well as deformations of the $\mathrm{C}-\mathrm{H}$ group. These vibrations have scarcely assigned to a specified functional group (Figure 4).

Contrary to the previous results, the wavenumber region of $1370-1230 \mathrm{~cm}^{-1}$ indicated the contribution of proteins (amide III) (Barth \& Zscherp, 2002), phenolic esters attachment at polysaccharides, and the group of methyl ester $\left(\mathrm{CH}_{3}\right)$ at another version of the report, which could come, for example, from the proteins and 4-O-glucuronic acids in ASG (Synytsya et al., 2003). A more sophisticated interpretation was required for the infrared spectral bands at the wavenumber region of 1200 and $800 \mathrm{~cm}^{-1}$, as it corresponded to ASG carbohydrate moiety (Figure 4). This particular section, also known as the carbohydrate fingerprint region, is produced by the side chains of polysaccharides and the skeletal stretching vibrations. It is also influenced by the ring vibrations that coincide with the stretching vibrations of $\mathrm{C}-\mathrm{O}-\mathrm{H}$ side groups and the vibrations of the C-O-C glycosidic bond.

The spectra of ASG from this wavenumber region were characterized against the other frequencies of Acacia gum samples, and the galactose and arabinose abundant polysaccharides (PRAGs) according to the methods reported by Boulet et al. (2007). The maximum ASG absorbance peaks established at 1027 and $1023 \mathrm{~cm}^{-1}$, and these results were in agreement with Lopez-Torrez et al. (2015), who performed an FTIR study towards raw Acacia senegal and Acacia seyal gum. In conclusion, the FTIR spectroscopy showed the ASG and PTC fingerprints with the main spectral variations by the composition of the glycosidic linkage.

However, the band comparison of the extractions and fractionated gum between the ASG and PTC showed that the resulting two CME FTIR spectra (Figure 4a and 5a) exhibited similar peaks position and shapes for certain specific bands to each other. Both ASG and PTC displayed the classic bioactive compounds absorptions at the approximated frequencies of 3741, 3684, 2915, 2361, 1635,1412 , and $1071 \mathrm{~cm}^{-1}$, similar to the mentioned peaks in the raw gum materials. The three peaks at each of the frequencies of $670,855,1071,782,1019$, and $3393 \mathrm{~cm}^{-1}$ signify the differences between the two extracts. The indication that there are more bioactive compound types in the CME compared to those in raw gum materials resulting from the data obtained and the different polarities of extraction solvents might cause this. Nevertheless, it is difficult to identify them unambiguously due to their tiny differences if there is only a conventional FTIR method.

Furthermore, the FTIR spectra also showed significant peaks of MF for both ASG and PTC, as shown in Figure 4 and Figure 5 (4a, c and 5b, c), where approximately fifteen peaks were lying between 418 to $3791 \mathrm{~cm}^{-1}$. Many differentiations on the FTIR spectra, which previously concealed from sight, now become apparent and more apparent, within the range of 2360 to $855 \mathrm{~cm}^{-1}$. Methanol fraction showed a higher number of absorption peaks compared to crude and acetone fraction (Figure $4 \mathbf{a}$ and 5a). For instance, the prominent peaks at 855, 1130, 1258, $1413,1492,1722,3023$, and $3484 \mathrm{~cm}^{-1}$ in MF were found to be absent from AF. Application to the peak's positions and intensities where the two fractions were found to be 
different in the range of 500 to $1770 \mathrm{~cm}^{-1}$. Moreover, the presence of ASG bioactive compounds found to changed regarding CME content and its $\mathrm{MF}$, with a range of 1880 to $614 \mathrm{~cm}^{-1}$. Hence, the enlarged fingerprint characters from CME and MF FTIR spectra can identify the two extracts quickly.

According to Figure 4a and 5a, the FTIR spectra, the ASG was different compared with the crude extract and fractions. For this reason, the CME of both samples validates the manifestation of an aliphatic amine, aromatic benzene, phenol (gallic acid), an alkane (Figure $4 a, b$, and $5 a, b)$, where the acetone fraction confirms the presence of alkanes and amine. The majority of phenolic phytoconstituents found from MF, such as ether, an aliphatic amine, carboxylic acid, alkanes, alkyl, and most of the phenolic compounds like gallic acid, tannic acid and p-Coumaric acid (Figure $4 a, b$, and $5 a, b$ ). Identical results were also reported from the work of Kannan et al. (2011).

Based on the observation of the FTIR spectra, the functional groups identified through the attribution of stretching and bending vibrations of the peaks. The peak areas (Figure $\mathbf{4 a}, \mathbf{b}$, and $\mathbf{5 a}, \mathbf{b}$ ) have identified in the IR domain, and the localization of the fingerprint region was carried out between 900 and $1500 \mathrm{~cm}^{-1}$. The first confinement of Area 1 with $<1000 \mathrm{~cm}^{-1}$ wavenumber corresponds to the $\mathrm{C}-\mathrm{H}$ isoprenoids bending vibrations, while the Area $2\left(997-1130 \mathrm{~cm}^{-1}\right)$ provides the C-O stretching vibrations of mono- and oligo-saccharides of carbohydrates, with signals at 1019, 1063, and $1068 \mathrm{~cm}^{-1}$. Area 3 (1150-1270 $\left.\mathrm{cm}^{-1}\right)$ corresponds to stretching vibrations of O-H or carbonyl C-O bindings, and Area 4 (1300-1450 $\mathrm{cm}^{-1}$ ) has a close similarity to the stretching vibrations of C-O (amide) and C-C from phenyl groups. Area 5 (within 1500-1600 $\mathrm{cm}^{-1}$ ) has related to $\mathrm{N}-\mathrm{H}$ bending vibrations and aromatic domain, while Area 6 is a multiplex structure (ranged from $1600-1760 \mathrm{~cm}^{-1}$ ), that corresponds to the bending waves of $\mathrm{N}-\mathrm{H}$ (amino acids) and $\mathrm{C}=\mathrm{O}$ stretchings (aldehydes and acetones, esters) at $1710 \mathrm{~cm}^{-1}$. Area 7 (2800 - $\left.2900 \mathrm{~cm}^{-1}\right)$, correlates with C-H stretching vibrations peculiar to $\mathrm{CH}_{3}$ and $\mathrm{CH}_{2}$ from lipids, C-H (aldehydes, including cis double bonds), and methoxy derivatives. The last cluster of Area 8 (3350-3600 $\mathrm{cm}^{-1}$ ) is consistent with the stretching vibrations of $\mathrm{OH}$ groups (e.g., water, phenols, alcohols, peroxides, and carbohydrates), as well as amides $\left(3650 \mathrm{~cm}^{-1}\right)$.

It is the first investigation that demonstrates the possible and efficient utilization of the FTIR technique for polyphenols measurement in GA extraction. The spectral differences were aimed at the objective to reflect the analyzed component variations. With the utilization of macroscopic fingerprint characters features from the FTIR spectrum, we can judge the origin of different extracts accurately and effectively, trace the constituents in the extracts, identify the authenticity of the medicinal materials, and even evaluate the qualities of bioactive substances.

The FTIR spectra are reflecting the perspective of chemical substances in an intricate system. For the identification of mix-substance systems, such as herbal and traditional medicines, it is still considered one of the most credible methods for the validation process. Therefore, the present work on ASG extracts exhibited novel phytochemical markers as a robust analytical tool not only to identify the essential bioactive compounds but also to examine the quality of the extract. Further investigations on advanced spectroscopic methods have needed for the identification and structural elucidation of specific phenol compounds. Finally, Figure 4 and Figure 5 showed the FTIR spectra for both ASG and PTC extract/fractions. 


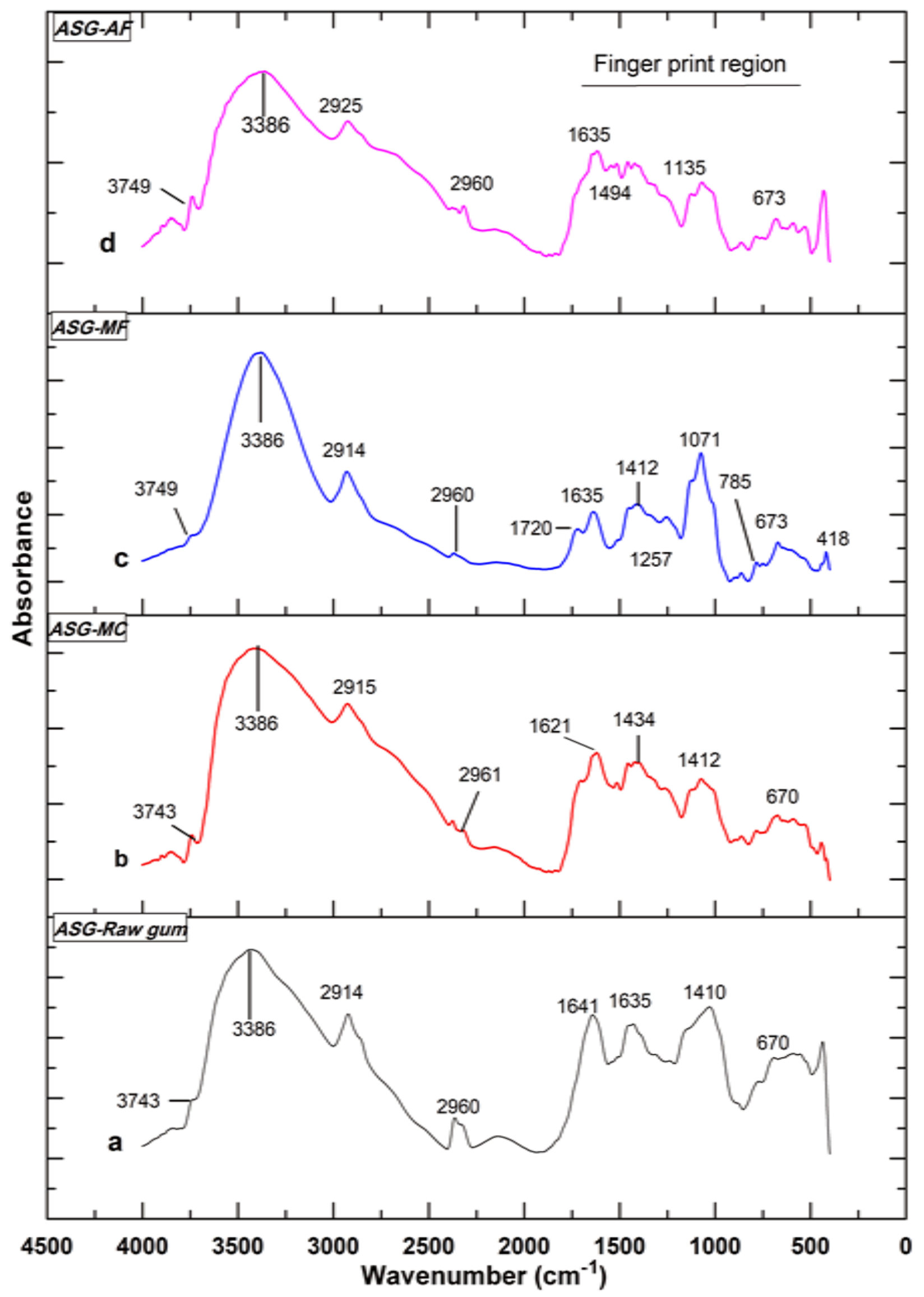

Figure 4. The FTIR spectra of ASG of raw gum (a), CME (b), MF (c), and AF (d), respectively. 


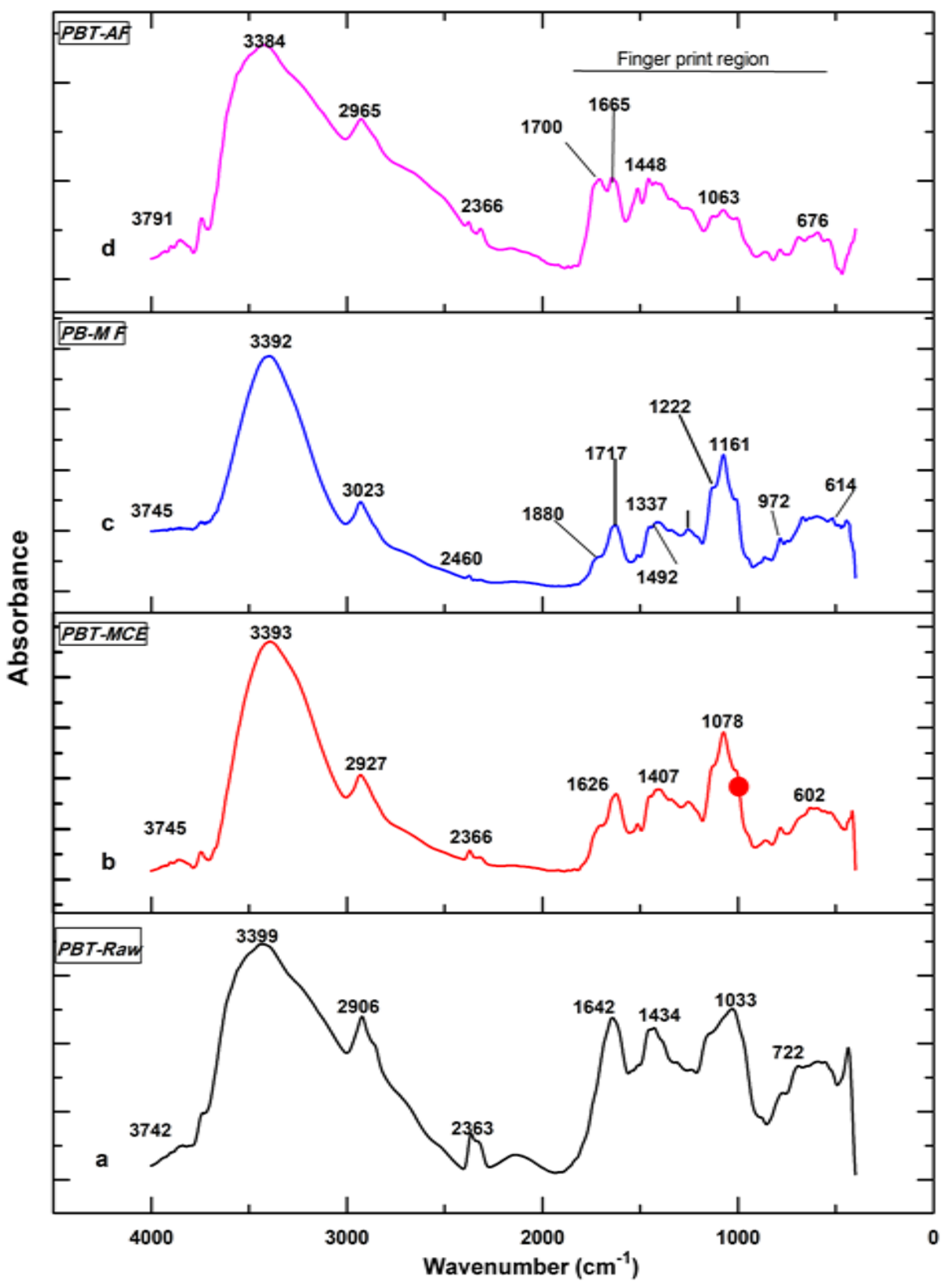

Figure 5. The FTIR spectra of PTC of raw gum (a), PTC CME (b), MF (c), and AF (d), respectively. 


\section{CONCLUSION}

In summary, this work can be considered as the first attempt to identify the biological potential of ASG. Based on the results, it has observed that the methanol-soluble fraction possesses the highest antioxidant activity of the $\mathrm{CME}$ and the different polarity fractions extracted from the ASG. Both MF and AF displayed the potential antioxidant capacities, respectively, which were secluded from CME by Kupchan solvent-solvent partitioning method. Both fractions showed maximum activity when using both CUPRAC and FRAP assays, with MF being the most active fraction. Through systematic analyses of FTIR spectroscopy towards CME and its different fractions, it has observed that the resulted spectra can deliver much information on macroscopic structures and comprehensive variation rules over the chemical constituents residing in the products. These methods can display the differences between the chemical components in CME and their different enriched fractions. The FTIR spectra can show more of the characteristics and features of the ASG samples, as the chemical compositions have concentrated after the extraction of the samples by solvents. The chemical substances in the extracts are somewhat consistent due to the commonality in the extraction methods, with the FTIR spectrum providing higher repeatability and comparability. Thus, the fact that the FTIR spectrum is capable of objectively reflecting the panorama of chemical constituents from a complex system made these methods have nominated as one of the most credible ways. The outcomes of this study contribute to the groundwork of designing future experimentation for a better comprehension of the antioxidative, anticancer, and anti-inflammatory effects of the gums. It may as well support the future work for discovering the phytochemicals associated with these properties.

\section{ACKNOWLEDGMENT}

The authors would like to express gratitude and gratefulness to the Department of Biotechnology Engineering (BTE), Faculty of Engineering, International Islamic University Malaysia (IIUM), and the International Institute for Halal Research and Training (INHART), IIUM for allowing using their laboratories and facilities. The acknowledgment extended to Dr. Elbasheir Sallam for his continuous and unlimited financial support to the Ph.D. candidate conducting this research

\section{REFERENCES}

Al-Mosawi, A.J. 2002. The etiology of chronic renal failure in 54 Iraqi children. Pediatric Nephrology. 17(6):463-464. https://doi.org/10.1007/s00467-001-0774-1

Ali, B.H., Al-Husseni, I., Beegam, S., Al-Shukaili, A., Nemmar, A., Schierling, S., Queisser, N., Schupp, N. 2013a. Effect of gum arabic on oxidative stress and inflammation in adenineinduced chronic renal failure in rats. PLoS One. 8(2):e55242. https://doi.org/10.1371/journal.pone.005524 2

Ali, B.H., Beegam, S., Al-Lawati, I., Waly, M.I., Al-Za'abi, M., Nemmar, A. 2013b. Comparative efficacy of three brands of gum acacia on adenineinduced chronic renal failure in rats. Physiological Research. 62(1):47-56.

Anderson, D.M.W. 1993. Some factors influencing the demand for gum arabic (Acacia senegal (L.) Wild.)_and other water-soluble tree exudates. Forest Ecology and Management. 58(1-2):1-18. https://doi.org/10.1016/0378-1127(93)901279

Apak, R., Güçlü, K., Özyürek, M., Çelik, S.E. 2008. Mechanism of antioxidant capacity assays and the CUPRAC (cupric ion reducing antioxidant capacity) assay. Microchimica Acta. 160(4):413419. https://doi.org/10.1007/s00604-0070777-0 
Barth, A., Zscherp, C. 2002. What vibrations tell about proteins. Quarterly Reviews of Biophysics. 35(4):369-430.

https://doi.org/10.1017/S0033583502003815

Boulet, J.C., Williams, P., Doco, T. 2007. A Fourier transform infrared spectroscopy study of wine polysaccharides. Carbohydrate Polymers. 69(1):79-85.

https://doi.org/10.1016/j.carbpol.2006.09.003

Chawla, R., Patil, G.R. 2010. Soluble Dietary Fiber. Comprehensive Reviews in Food Science and Food Safety. 9(2):178-196. https://doi.org/10.1111/j.15414337.2009.00099.x

Doi, Y., Ichihara, T., Hagiwara, A., Imai N., Tamano, S., Orikoshi, H., Ogasawara, K., Sasaki, Y., Nakamura, M., Shirai, T. 2006. A ninety-day oral toxicity study of a new type of processed gum arabic, from Acacia tree (Acacia senegal) exudates, in F344 rats. Food and Chemical Toxicology. $\quad$ 44(4):560-566. https://doi.org/10.1016/j.fct.2005.09.002

Elnour, A.A.M. 2007. Fractionation; Physicochemical and Functional properties of Acacia Polyacantha gum. Thesis. University of Khartoum, Sudan.

Elnour, A.A.M., Mirghani, M.E.S., Kabbashi, N.A., Alam, M.Z., Musa, K.H. 2018. Study of Antioxidant and Anti-Inflammatory Crude Methanol Extract and Fractions of Acacia seyal Gum. American Journal of Pharmacology and Pharmacotherapeutics. 5(1):3. https://doi.org/10.21767/2393-8862.100012

Glover, D.A., Ushida, K., Phillips, A.O., Riley, S.G. 2009. Acacia(sen) SUPERGUM ${ }^{\mathrm{TM}}$ (Gum arabic): An evaluation of potential health benefits in human subjects. Food Hydrocolloids. 23(8):24102415.

https://doi.org/10.1016/j.foodhyd.2009.06.02 0

Godic, A., Poljšak, B., Adamic, M., Dahmane, R. 2014. The Role of Antioxidants in Skin Cancer Prevention and Treatment. Oxidative Medicine and Cellular Longetivity. 2014:860479. https://dx.doi.org/10.1155/2014/860479

Jayasri, D., Narayanan, S.S. 2007. Manganese(II) hexacyanoferrate based renewable amperometric sensor for the determination of butylated hydroxyanisole in food products.
Food Chemistry. 101(2):607-614. https://doi.org/10.1016/j.foodchem.2006.02. 021

Kaewseejan, N., Sutthikhum, V., Siriamornpun, S. 2015. Potential of Gynura procumbens leaves as source of flavonoid-enriched fractions with enhanced antioxidant capacity. Journal of Functional Foods. 12:120-128. https://doi.org/10.1016/j.jff.2014.11.001

Kannan, R.R.R., Arumugam, R., Anantharaman, P. 2011. Fourier Transform Infrared Spectroscopy Analysis of Seagrass Polyphenols. Current Bioactive Compounds. 7(2):118-125. https://doi.org/10.2174/15734071179601114 2

Kupchan, S.M., Steyn, P.S., Grove, M.D., Horsfield, S.M., Meitner, S.W. 1969. Tumor inhibitors. XXXV. Myrsine saponin, the active principle of Myrsine africana L. Journal of Medicinal Chemistry. 12(1):167-169. https://doi.org/10.1021/jm00301a045

Lebon, M., Zazzo, A., Reiche, I. 2014. Screening in situ bone and teeth preservation by ATR-FTIR mapping. Palaeogeography, Palaeoclimatology, Palaeoecology.

416:110-119. https://doi.org/10.1016/j.palaeo.2014.08.001

Lopez-Torrez, L., Nigen, M., Williams, P., Doco, T., Sanchez, C. 2015. Acacia senegal vs. Acacia seyal gums - Part 1: Composition and structure of hyperbranched plant exudates. Food Hydrocolloids. 51:41-53. https://doi.org/10.1016/j.foodhyd.2015.04.01 9

Magid, T.D.A., Eltayb, M.T.A., Dirar, A.M.A. 2014. Equalizing Gum Codal Term (a Code E414), of Acacia Senega1 (L) with Acacia Seyal (Del.), and its Impact on Gum Production and Exportation. Journal of Applied and Industrial Sciences. 2(3):144-151.

Manrique, G.D., Lajolo, F.M. 2002. FT-IR spectroscopy as a tool for measuring degree of methyl esterification in pectins isolated from ripening papaya fruit. Postharvest Biology and Technology. 25(1):99-107. https://doi.org/10.1016/S09255214(01)00160-0

Minzanova, S.T., Mironov, V.F., Arhipova, D.M., Khabibullina, A.V., Mironova, L.G., Zakirova, Y.M., Milyukov, V.A. 2018. Biological Activity 
and Pharmacological Application of Pectic Polysaccharides: A Review. Polymers. 10(12):1407.

https://dx.doi.org/10.3390/polym10121407

Musa, H.H., Ahmed, A.A., Musa, T.H., Fedail, J.S. 2015. Gum arabic down-regulate PPAR-y and SCD mRNA expression in mice. Polish Annals of Medicine. 22(1):11-17. https://doi.org/10.1016/j.poamed.2015.03.00 8

Musa, K.H., Abdullah, A., Kuswandi B., Hidayat, M.A. 2013. A novel high throughput method based on the DPPH dry reagent array for determination of antioxidant activity. Food Chemistry. 141(4):4102-4106. https://doi.org/10.1016/j.foodchem.2013.06. 112

Niu, F., Niu, D., Zhang, H., Chang, C., Gu, L., Su, Y., Yang, Y. 2016. Ovalbumin/gum arabicstabilized emulsion: Rheology, emulsion characteristics, and Raman spectroscopic study. Food Hydrocolloids. 52:607-614. https://doi.org/10.1016/j.foodhyd.2015.08.01 0

Ouhaddouch, H., Cheikh, A., Idrissi, M.O.B., Draoui, M., Boutia, M. 2019. FT-IR Spectroscopy Applied for Identification of a Mineral Drug Substance in Drug Products: Application to Bentonite. Journal of Spectroscopy. 2019:2960845. https://doi.org/10.1155/2019/2960845

Sanchez, C., Nigen, M., Tamayo, V.M., Doco, T., Williams, P., Amine, C., Renard, D. 2018. Acacia gum: History of the future. Food Hydrocolloids.

78:140-160. https://doi.org/10.1016/j.foodhyd.2017.04.00 8

Rehman, K.U., Wingertzahn, M.A., Teichberg, S., Harper, R.G., Wapnir, R.A. 2003. Gum arabic (GA) modifies paracellular water and electrolyte transport in the small intestine. Digestive Diseases and Sciences. 48(4):755-760. https://doi.org/10.1023/a:1022845011192

Reinholds, I., Bartkevics, V., Silvis, I.C.J., van Ruth, S.M., Esslinger, S. 2015. Analytical techniques combined with chemometrics for authentication and determination of contaminants in condiments: A review. Journal of Food Composition and Analysis. 44:56-72. https://doi.org/10.1016/j.jfca.2015.05.004
Renard, D., Lavenant-Gourgeon, L., Ralet, M.C., Sanchez, C. 2006. Acacia senegal gum: continuum of molecular species differing by their protein to sugar ratio, molecular weight, and charges. Biomacromolecules. 7(9):2637-2649. https://doi.org/10.1021/bm060145j

Synytsya, A., Čopıkováa, J., Matějkab, P., Machovič, V. 2003. Fourier transform Raman and infrared spectroscopy of pectins. Carbohydrate Polymers. 54(1):97-106. https://doi.org/10.1016/S01448617(03)00158-9

Tagne, R.S., Telefo, B.P., Nyemb, J.N., Yemele, D.M., Njina, S.N., Goka, S.M., Lienou, L.L., NwaboKamdje, A.H., Moundipa, P.F., Farooq, A.D. 2014. Anticancer and antioxidant activities of methanol extracts and fractions of some Cameroonian medicinal plants. Asian Pacific Journal of Tropical Medicine. 7S1:S442-S447. https://doi.org/10.1016/S19957645(14)60272-8

Tiss, A., Carrière, F., Verger, R. 2001. Effects of Gum Arabic on Lipase Interfacial Binding and Activity. Analytical Biochemistry. 294(1):36-43. https://doi.org/10.1006/abio.2001.5095

Vinod, V.T.P., Sashidar, R.B., Sarma, V.U.M., Raju, S.S. 2010. Comparative amino acid and fatty acid compositions of edible gums kondagogu (Cochlospermum gossypium) and karaya (Sterculia urens). Food Chemistry. 123(1):57-62. https://doi.org/10.1016/j.foodchem.2010.03. 127

Wang, H., Williams, P.A., Senan, C. 2014. Synthesis, characterization and emulsification properties of dodecenyl succinic anhydride derivatives of gum Arabic. Food Hydrocolloids. 37:143-148. https://doi.org/10.1016/j.foodhyd.2013.10.03 3 